\title{
Design of RCPC Encoded V-BLAST MIMO System
}

\author{
Lydia Sari $^{1}$, Gunawan Wibisono ${ }^{2} \&$ Dadang Gunawan $^{3}$ \\ ${ }^{1,2,3}$ Electrical Engineering Department, Faculty of Engineering \\ University of Indonesia \\ 1'lydia.sari@ui.edu, ${ }^{2}$ gunawan@eng.ui.ac.id, ${ }^{3}$ guna@eng.ui.ac.id
}

\begin{abstract}
A Vertical Bell Laboratories Layered Space-Time Multiple-Input Multiple Output (V-BLAST MIMO) enhanced with Unequal Error Protection (UEP) to achieve highly reliable wireless communication is proposed. The UEP scheme is based on Channel State Information (CSI) available at the transmitter whose calculation utilizes Singular Value Decomposition (SVD) of the MIMO matrix channel. Using Rate-Compatible Punctured Convolutional (RCPC), a different code rate is given for each sub-stream of source information, according to its level of transmit power. To analyze the system performance, an analytical BER comprising the performance of V-BLAST MIMO BPSK-modulated signals and the performance of RCPC codes in Rayleigh fading environment is presented. Simulation results show that increasing the code rate can attain a bandwidth efficiency of $33.3 \%$ in expense $E_{b} / N_{\mathrm{o}}$, but this penalty is not severe as the high code rate is used in sub-channels with high attenuation level. It is also shown that a system with 2 transmit and 4 receive antennas will have an improved performance within only $1 \mathrm{~dB}$ range compared to a system with 2 transmit and 2 receive antennas. The performance of the proposed system is mostly affected by the type of puncturing matrices chosen.
\end{abstract}

Keywords: BER; MIMO; RCPC code; singular value decomposition; V-BLAST.

\section{Introduction}

The multiple-input multiple-output (MIMO) wireless communication scheme, characterized with multiple antennas in both the transmitter and receiver sides, has gained a lot of interest in the past decade. Its potential of realizing enormous capacity without necessitating extra bandwidth is especially appealing for today's multimedia communication needs [1-3]. The high capacity nature of the MIMO scheme is attained by exploiting multipath scattering, therefore the scheme suits an indoor environment where a rich scattering pattern exists.

A proper processing architecture is crucial to achieve this high capacity potential. Vertical Bell laboratories layered space-time (V-BLAST) is one of such architectures known to have spectral efficiencies of $20-40 \mathrm{bps} / \mathrm{Hz}$ at $24-34$ $\mathrm{dB}$ average SNR without coding [2]. It is noted however that an appropriate coding scheme is required to complement the high data rate of the system with a high diversity gain.

Received September $15^{\text {th }}, 2009$. 
A coding approach in which diversity gain and multiplexing gain are taken into consideration is unequal error protection (UEP). The basic idea of this approach is to assign different levels of error protection to different data streams. By providing different levels of error protection, a high data redundancy is mitigated. This will in turn contribute to bandwidth efficiency. UEP can be attained using rate-compatible punctured convolutional (RCPC) codes, in which a single encoder and decoder structure is employed to provide different code rates, hence giving different levels of protection to the information source [4]. Several researches have proposed the use of UEP with MIMO scheme [5-8]. The UEP proposed in these researches imply the need to resend information repeatedly using progressively stronger code protections, and therefore are more suitable for systems requiring automatic repeat request (ARQ). For wireless system communications where ARQ is typically not implemented, there is a need for a different way to implement UEP.

It is shown in our previous research on UEP and V-BLAST MIMO that an RCPC-encoded V-BLAST MIMO is capable of achieving consistently better performance in respect to a lowered code rate [9-10]. In these works, however, the modulation used was QAM, which do not allow for accurate separation between the signal and the fading amplitudes. As a first consequence, the theoretical BER presented therein is an average for an assumed equal transmit power scheme. In [9], the power is assumed to be constant for all transmit antennas, while in [10] it is calculated as a function of channel gains and the number of antennas. The second consequence is that in these works an allocation scheme for different code rates for different sub-channels in the MIMO system are not addressed. The RCPC codes used in [9-10] are taken from [4], although clearly alternate RCPC codes can be built for different puncturing periods.

This paper intends to address the above issues by BPSK modulation so that the signal amplitude is constant and the fading amplitude can be observed more accurately. This consequently allows the use of differently allocated power and code rates for different transmit antennas. New RCPC codes with puncturing periods $L=5$ and 6 are also presented. As opposed to applying different code rates to different data streams according to their level of urgency, in this paper we use Channel State Information (CSI) at the transmitter to first differentiate the transmit powers given to the data streams transmitted by the multiple antennas. A data stream entering a sub-channel with low level attenuation will be given a higher transmit power than a data stream entering a sub-channel with high level of attenuation. Different code rates are subsequently assigned according to the allocated transmit power. A high level of transmit power will be paired with a low code rate, meaning the information sub-stream entering a sub-channel with low level attenuation will benefit from both high transmit 
power and high level error protection. The proposed system uses $m$ transmit and $n$ receive antennas. An analytical BER comprising the performance of $\mathrm{V}$ BLAST MIMO BPSK-modulated signals and the performance of RCPC codes in Rayleigh fading environment is presented and used to analyze the system performance.

This paper is organized as follows. Section 2 gives the system model covering the RCPC code design, the V-BLAST MIMO design with CSI and the system performance. Simulation results are discussed in Section 3 and the conclusion is given in Section 4.

\section{$2 \quad$ System Model}

\subsection{RCPC Code Design}

The proposed RCPC-encoded V-BLAST MIMO with UEP system model is given in Figure 1. The channel is assumed to undergo Rayleigh fading and there exists a rich scattering paths between the transmit and receive antennas. The transmitter block consists of a V-BLAST demodulator and an RCPC encoder.

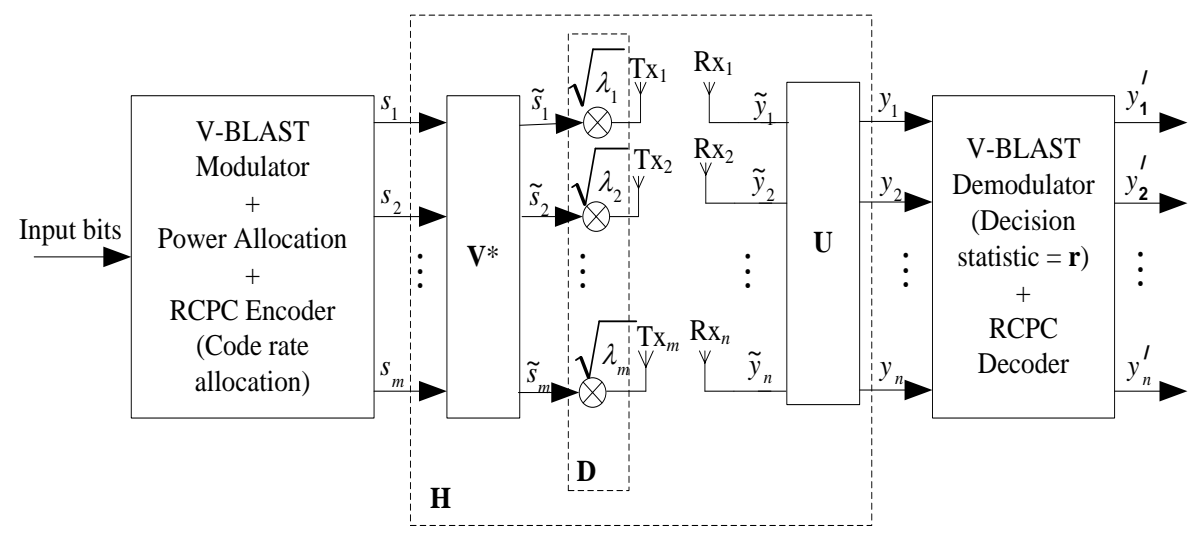

Figure 1 System model.

The RCPC encoder enables the assignment of RCPC codes with rates $R c_{i}$, $i=1, . ., m$ to the data streams according to the sub-channel gains. A low rate code is given to a data stream entering a sub-channel with low attenuation, and vice versa. This is intended to provide high level of protection only to the data stream entering a non-destructive sub-channel. 
The different convolutional code rates are derived from a mother code with rate $1 / N$. For a puncturing period of $L$ bits, the resulting punctured convolutional code rates are [5]

$$
R_{c_{i}}=\frac{L}{L+\delta} \quad, \delta=1, \ldots,(N-1) L, i=1, \ldots, m
$$

The puncturing matrix used is [4]

$$
a(\delta)=\left[\begin{array}{ccc}
a_{11} & \ldots & a_{1 j} \\
\vdots & \ddots & \vdots \\
a_{i 1} & \cdots & a_{i j}
\end{array}\right] \quad i=1, \ldots, N, j=1, \ldots, L
$$

which consists of $N$ rows and $L$ columns, and $a_{i j}(\delta) \in(0,1)$ in which 0 implies a puncturing. The number of possibilities to form the puncturing matrix $a_{i j}(\delta)$ is

$$
\frac{1}{N} \cdot\left(\begin{array}{c}
N L \\
\ell
\end{array}\right)
$$

where $\ell$ denotes the number of zeros in the puncturing matrix. The division by factor $N$ is due to the fact that a cyclic shift of $N$ symbols in the puncturing matrix will give the same performance with the case of no cyclic shift. In the first simulation the parameters used are $N=3, R_{c 1}=5 / 15$ and $R_{c 2}=5 / 10$ for two transmitted data streams with $L=5$. In the second simulation the parameters used are $N=3, R_{c 1}=6 / 18$ and $R_{c 2}=6 / 12$ for two transmitted data streams with $L$ $=6$. Figure 2 shows an example of the RCPC encoder of the proposed system model.

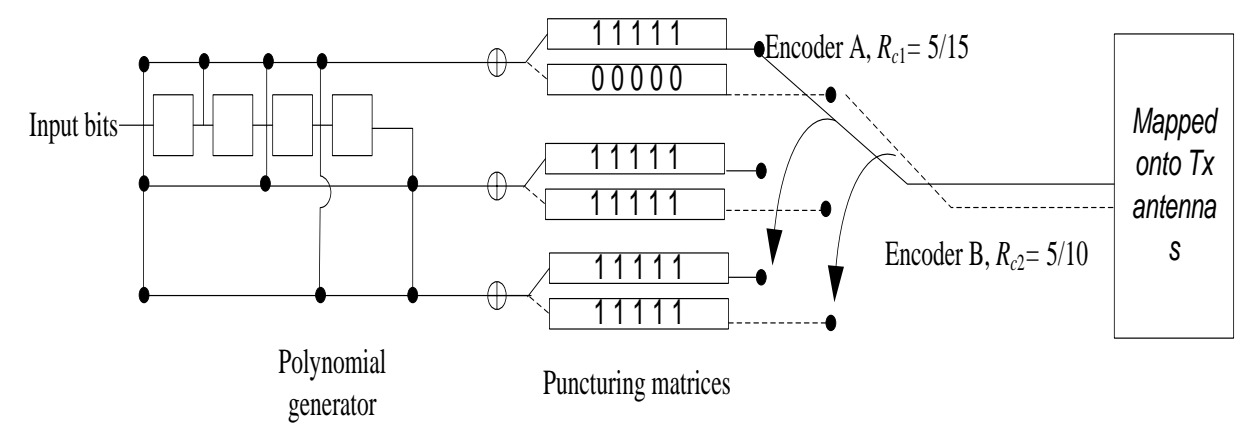

Figure 2 Example of RCPC encoder with mother code generator polynomial [36 25 23] and puncturing matrices from encoders A and B depicted in Table 1. 
There are no systematic methods available to construct good rate $L / L+\delta$ codes [4]. Consequently, the search for good codes in this paper is based on the bestknown generator polynomial of rate $1 / 3$. This generator polynomial yields a "mother code". The bits in the mother code are then punctured to attain codes of rates $1 / 2$, using different puncturing matrices.

For the case of $L=5$, the mother code rate is $5 / 15$ with generator polynomial [ 362523 ] and the puncturing matrix is shown in Table 1 as encoder A. The minimal Hamming distance between different codewords, termed free distance or $d_{f r e e}$ for this encoder is 10 . Therefore the encoder does not introduce error weight $c_{d}$ in $d_{\text {free }}<10$. However for several values of $d_{\text {free }}$ the error weight $c_{d}$ equals 0 . This is due to the fact that the Hamming distances from the resulting codewords and the all-zero paths are even, therefore for odd values of $d_{\text {free }}$, there is no $c_{d}$ found.

Table 1 Puncturing Matrices $a(\delta)$ and $c_{d}$ Values for RCPC Codes with Puncturing Period $L=5$ and Memory $M=5$

\begin{tabular}{|c|c|c|c|c|c|c|c|c|c|c|c|c|c|}
\hline \multicolumn{2}{|c|}{ Encoder } & & 6 & 7 & 8 & 9 & 10 & 11 & 12 & 13 & 14 & 15 & 16 \\
\hline A & $\begin{array}{l}\text { - } R c_{1}=5 / 10 \\
\text { - Puncturing matrix } \\
a(10)=\left[\begin{array}{lllll}1 & 1 & 1 & 1 & 1 \\
1 & 1 & 1 & 1 & 1 \\
1 & 1 & 1 & 1 & 1\end{array}\right] \\
\text { - Generator } \\
\text { polynomial [36 } 25 \\
\text { 23] }\end{array}$ & $c_{d}$ & & & & & 1 & 0 & 8 & 0 & 30 & 0 & 100 \\
\hline B & $\begin{array}{l}\text { - } R c_{1}=5 / 10 \\
\text { - Puncturing matrix } \\
a(10)=\left[\begin{array}{llllll}0 & 0 & 0 & 0 & 0 \\
1 & 1 & 1 & 1 & 1 \\
1 & 1 & 1 & 1 & 1\end{array}\right] \\
\text { - Generator } \\
\text { polynomial }\left[\begin{array}{lll}00 & 25 \\
23\end{array}\right]\end{array}$ & $c_{d}$ & 1 & 0 & 18 & 0 & 53 & 0 & 1088 & 0 & 7384 & 0 & 47734 \\
\hline $\mathrm{C}$ & $\begin{array}{l}\text { - } R c_{1}=5 / 10 \\
\text { - Puncturing matrix } \\
a(10)=\left[\begin{array}{llllll}1 & 1 & 1 & 1 & 1 \\
0 & 0 & 0 & 0 & 0 \\
1 & 1 & 1 & 1 & 1\end{array}\right] \\
\text { - Generator } \\
\text { polynomial }\left[\begin{array}{lll}36 & 00 \\
23\end{array}\right]\end{array}$ & $c_{d}$ & 2 & 4 & 8 & 46 & 142 & 313 & 726 & 1864 & 4870 & 12217 & 29890 \\
\hline D & - $R c_{1}=5 / 10$ & $c_{d}$ & 2 & 4 & 10 & 19 & 80 & 240 & 634 & 1583 & 4128 & 10621 & 26792 \\
\hline
\end{tabular}




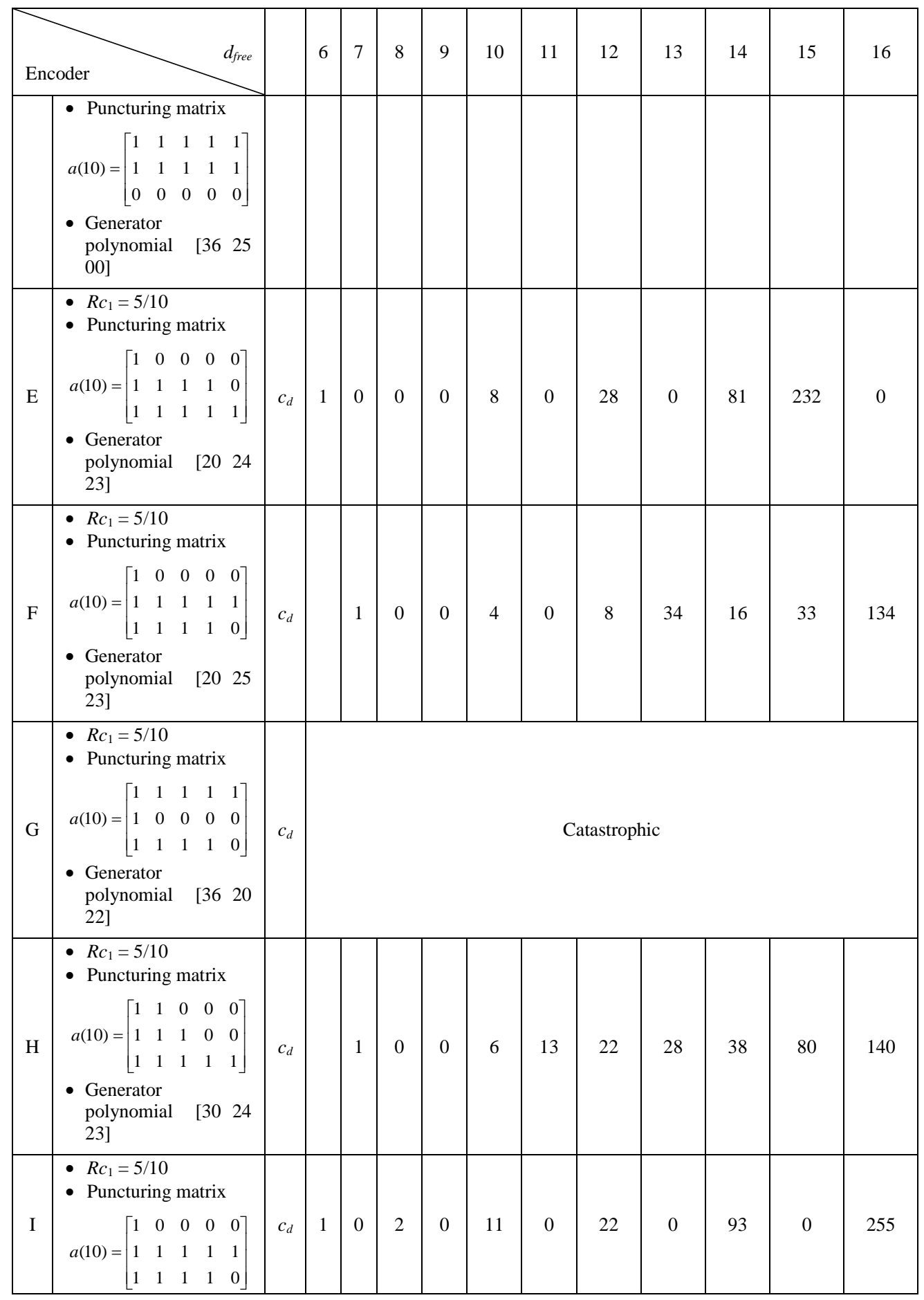




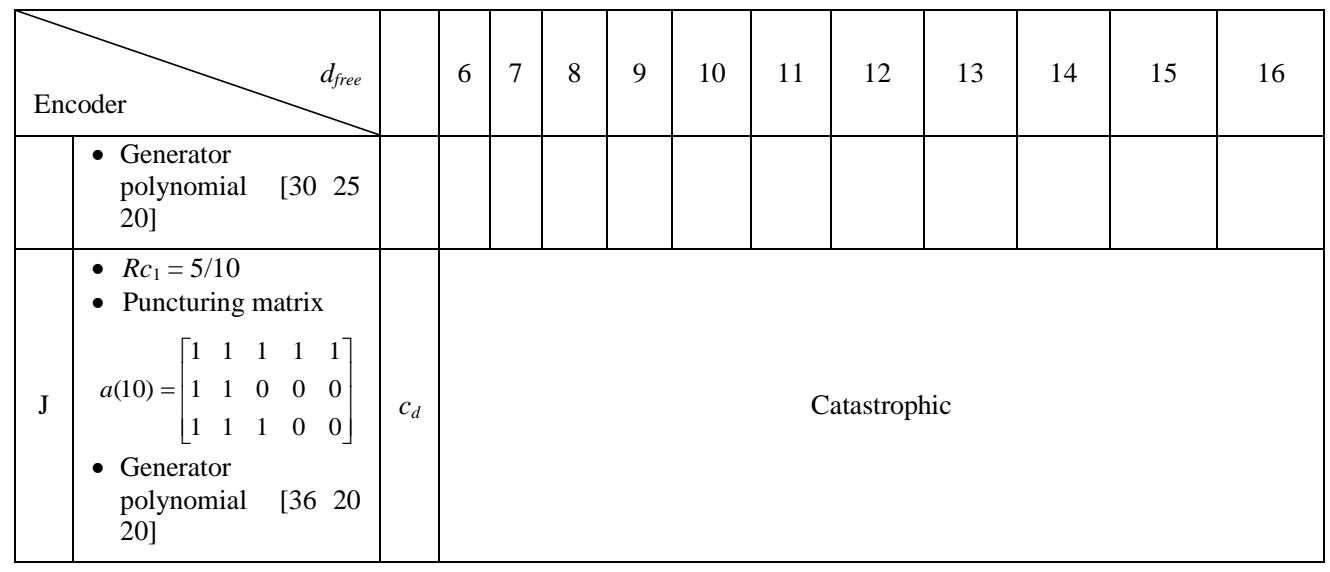

To yield codes with rates $5 / 10$ from this mother code, there are 5 bits that need to be punctured. As stated earlier there are many possibilities as to which 5 bits can be punctured. For simplicity reason, in the first three simulations the 5 punctured bits are all in the same row. As calculating the error weights $c_{d}$ in the incorrect paths of a convolutional code require extensive computer search, in this paper we use a polynomial generator equivalents to simplify the calculation. For example, for the mother code [ 362523 ], the generator polynomial in binary form is

$$
\left[\begin{array}{lllll}
1 & 1 & 1 & 1 & 0 \\
1 & 0 & 1 & 0 & 1 \\
1 & 0 & 0 & 1 & 1
\end{array}\right]
$$

where ' 1 ' denotes a connection between a memory register and the corresponding summing points of the convolutional encoder, while ' 0 ' denotes none of such connection. In encoder B depicted in Table 1, all of the bits contained in the codeword yielded by the first summing point are punctured. This is equal to removing all of the connections between the memory registers and the first summing point in the encoder, so that the equivalent generator polynomial is

$$
\left[\begin{array}{lllll}
0 & 0 & 0 & 0 & 0 \\
1 & 0 & 1 & 0 & 1 \\
1 & 0 & 0 & 1 & 1
\end{array}\right]
$$

In octal form, the above matrix can be stated as [00 25 23]. Using this equivalent generator polynomial, the free distance $d_{\text {free }}$ and error weights $c_{d}$ can 
be calculated. The resulting $d_{\text {free }}$ and $c_{d}$ values for different puncturing matrices with $L=5$ and memory $M=5$ are given in Table 1 . It is shown that several puncturing matrices will yield catastrophic codes, as these matrices do not provide an invertible feed-forward generator polynomial. Moreover, some puncturing matrices will yield codes with $M<5$ and therefore are not included in the table.

The parameters for a mother code with rate $6 / 18$ are $L=6$ and generator polynomial [ 7553 47] as depicted in Table 2 . To yield codes with rates 6/12 from this mother code, 6 bits of the codeword will be punctured. Again in the first three simulations, the 6 punctured bits are in one row, while in the subsequent simulations the punctured bits are located in more than one row. The mother code generator polynomial [75 53 47] can be stated in binary form as

$$
\left[\begin{array}{llllll}
1 & 1 & 1 & 1 & 0 & 1 \\
1 & 0 & 1 & 0 & 1 & 1 \\
1 & 0 & 0 & 1 & 1 & 1
\end{array}\right]
$$

Similar with the previous definition, ' 1 ' denotes a connection between a memory register and the corresponding summing points of the convolutional encoder, while ' 0 ' denotes none of such connection. In Encoder $\mathrm{O}$ of Table 2, three bits each in the first and the second row are punctured. The positions of the punctured bits are in the right-most of the matrices, i.e. the least significant bits. Puncturing the three least significant bits is equal to eliminating the connections of the last three memory registers with the corresponding summing points, yielding an equivalent generator polynomial in the form of

$$
\left[\begin{array}{llllll}
1 & 1 & 1 & 1 & 0 & 1 \\
1 & 0 & 1 & 0 & 1 & 1 \\
1 & 0 & 0 & 1 & 1 & 1
\end{array}\right] \rightarrow\left[\begin{array}{llllll}
1 & 1 & 1 & 0 & 0 & 0 \\
1 & 0 & 1 & 0 & 0 & 0 \\
1 & 0 & 0 & 1 & 1 & 1
\end{array}\right]
$$

In octal form, the matrix in the right of the equation sign above can be stated as [70 50 47]. The values of $d_{\text {free }}$ and $c_{d}$ are computed using this equivalent generator polynomial. Proceeding with other possible puncturing matrices, $d_{\text {free }}$ and $c_{d}$ are computed and are given in Table 2. As with the case of $L=5$, several puncturing matrices will yield catastrophic codes, and some others will yield codes with $M<5$ and therefore are omitted from the table.

The computations of $d_{f r e e}$ and $c_{d}$ are limited to $d_{f r e e}=16$ as this number already represents the performance of the code with greater $d_{\text {free }}$. Simulations with greater $d_{\text {free }}$ have been done for this research, and it is found that greater $d_{\text {free }}$ 
values do not only require extensive resources but also do not significantly affect the outcomes of the results shown in Section 3.

Tables 1 and 2 show that puncturing the data stream will shorten the free distance of the codes. Nevertheless, by increasing the puncturing period $L$ better codes can be achieved. As RCPC codes introduce long decision depths and $N L$ ambiguity to be resolved in the incoming data stream, a proper termination or synchronization will be needed in systems that use this code.

Table 2 Puncturing Matrices $a(\delta)$ and $c_{d}$ Values for RCPC Codes with Memory $\mathrm{M}=5$ and Puncturing Period $\mathrm{L}=6$.

\begin{tabular}{|c|c|c|c|c|c|c|c|c|c|c|c|c|}
\hline & ${ }_{\text {oder }} d_{\text {free }}$ & & 7 & 8 & 9 & 10 & 11 & 12 & 13 & 14 & 15 & 16 \\
\hline K & $\begin{array}{l}\text { - } R c_{1}=6 / 18 \\
\text { - Puncturing matrix } \\
a(18)=\left[\begin{array}{llllll}1 & 1 & 1 & 1 & 1 & 1 \\
1 & 1 & 1 & 1 & 1 & 1 \\
1 & 1 & 1 & 1 & 1 & 1\end{array}\right] \\
\text { - Generator polynomial } \\
\text { [75 53 47] }\end{array}$ & $c_{d}$ & & & & & & & 1 & 8 & 26 & 20 \\
\hline L & $\begin{array}{l}\text { - } R c_{2}=6 / 12 \\
\text { - Puncturing matrix } \\
a(12)=\left[\begin{array}{llllll}0 & 0 & 0 & 0 & 0 & 0 \\
1 & 1 & 1 & 1 & 1 & 1 \\
1 & 1 & 1 & 1 & 1 & 1\end{array}\right] \\
\text { - Generator polynomial } \\
\text { [0 53 47] }\end{array}$ & $c_{d}$ & & & & & & & astrop & & & \\
\hline M & $\begin{array}{l}\text { - } R c_{2}=6 / 12 \\
a(12)=\left[\begin{array}{llllll}1 & 1 & 1 & 1 & 1 & 1 \\
0 & 0 & 0 & 0 & 0 & 0 \\
1 & 1 & 1 & 1 & 1 & 1\end{array}\right] \\
\text { Generator polynomial } \\
\text { [75 0 47] }\end{array}$ & $c_{d}$ & 3 & 2 & 1 & 52 & 111 & 236 & 735 & 1842 & 4933 & 13174 \\
\hline $\mathrm{N}$ & $\begin{array}{l}\text { - } R c_{2}=6 / 12 \\
a(12)=\left[\begin{array}{llllll}1 & 1 & 1 & 1 & 1 & 1 \\
1 & 1 & 1 & 1 & 1 & 1 \\
0 & 0 & 0 & 0 & 0 & 0\end{array}\right] \\
\text { - Guncturing matrix } \\
{[75530]}\end{array}$ & $c d$ & & 2 & 36 & 32 & 62 & 332 & 701 & 2342 & 5503 & 12506 \\
\hline $\mathrm{O}$ & $\begin{array}{l}\text { - } R c_{2}=6 / 12 \\
\text { - Puncturing matrix }\end{array}$ & $c_{d}$ & & 1 & 2 & 6 & 10 & 6 & 32 & 73 & 74 & 147 \\
\hline
\end{tabular}




\begin{tabular}{|c|c|c|c|c|c|c|c|c|c|c|c|c|}
\hline \multicolumn{2}{|c|}{ Encoder } & & 7 & 8 & 9 & 10 & 11 & 12 & 13 & 14 & 15 & 16 \\
\hline & $\begin{array}{l}a(12)=\left[\begin{array}{llllll}1 & 1 & 1 & 0 & 0 & 0 \\
1 & 1 & 1 & 0 & 0 & 0 \\
1 & 1 & 1 & 1 & 1 & 1\end{array}\right] \\
\text { - Generator polynomial } \\
{[7050 \text { 47] }}\end{array}$ & & & & & & & & & & & \\
\hline $\mathrm{P}$ & $\begin{array}{l}\text { - } R c_{2}=6 / 12 \\
\text { - Puncturing matrix } \\
a(12)=\left[\begin{array}{llllll}1 & 1 & 1 & 0 & 0 & 0 \\
1 & 1 & 1 & 1 & 1 & 1 \\
1 & 1 & 1 & 0 & 0 & 0\end{array}\right] \\
\text { - Generator polynomial } \\
\text { [70 53 40] }\end{array}$ & $c_{d}$ & & 1 & 0 & 10 & 0 & 21 & 0 & 57 & 0 & 232 \\
\hline Q & $\begin{array}{l}\text { - } R c_{2}=6 / 12 \\
a(12)=\left[\begin{array}{llllll}1 & 1 & 1 & 1 & 1 & 1 \\
1 & 1 & 1 & 0 & 0 & 0 \\
1 & 1 & 1 & 0 & 0 & 0\end{array}\right] \\
\text { - Generator polynomial } \\
\text { [75 50 40] }\end{array}$ & $c_{d}$ & & 3 & 0 & 8 & 0 & 21 & 0 & 96 & 0 & 223 \\
\hline $\mathrm{R}$ & $\begin{array}{l}\text { - } R c_{2}=6 / 12 \\
\text { - Puncturing matrix } \\
a(12)=\left[\begin{array}{llllll}1 & 1 & 1 & 1 & 0 & 0 \\
1 & 1 & 1 & 1 & 0 & 0 \\
1 & 1 & 1 & 1 & 0 & 0\end{array}\right] \\
\text { - Generator polynomial } \\
\text { [74 50 44] }\end{array}$ & $c_{d}$ & & & & & & & stro & & & \\
\hline$S$ & $\begin{array}{l}\text { - } R c_{2}=6 / 12 \\
\text { - Puncturing matrix } \\
a(12)=\left[\begin{array}{llllll}1 & 1 & 1 & 0 & 0 & 1 \\
1 & 1 & 1 & 0 & 0 & 1 \\
1 & 1 & 1 & 0 & 0 & 1\end{array}\right] \\
\text { - Generator polynomial } \\
\text { [71 51 41] }\end{array}$ & $c_{d}$ & & & 1 & 0 & 0 & 2 & 3 & 10 & 12 & 20 \\
\hline
\end{tabular}

\subsection{V-BLAST MIMO Model with CSI}

In the proposed system model, CSI is fed onto the transmitter block so that different power levels can be allocated to different transmit antennas, according to the channel condition. The V-BLAST modulator in the transmitter block is basically a demultiplexer, which is used to map a single stream of information bits onto the multiple transmit antennas. BPSK modulation is chosen for this system, so that every symbol transmitted consists of one information bit. The elements of a signal vector $\mathbf{s}=\left[s_{1}, s_{2}, \ldots, s_{m}\right]^{T}$ are transmitted simultaneously 
from first to the $m$-th transmit antennas, and the signal arriving at the receive antennas $\mathbf{y}=\left[y_{1}, y_{2}, \ldots, y_{n}\right]^{T}$ can be expressed as

$$
\mathbf{y}=\mathbf{H s}+\mathbf{n}
$$

where $\mathbf{H}$ is the matrix channel of a MIMO system whose elements are the channel gains between the transmit and receive antennas, and $\mathbf{n}$ is a noise vector with complex Gaussian distribution, zero mean and variance $\sigma^{2}$.

One way to make the matrix channel known at the transmitter is by using Singular Value Decomposition (SVD). Using SVD, the matrix channel $\mathbf{H}$ is decomposed into [3]

$$
\mathbf{H}=\mathbf{U} \cdot \mathbf{D} \cdot \mathbf{V}^{*}
$$

where $\mathbf{U}$ and $\mathbf{V}$ are complex unitary matrices which dimensions are $m \times m$ and $n \times n$ respectively, (.) ${ }^{*}$ denotes conjugate transpose and $\mathbf{D}$ is diagonal matrix whose dimension is $m \times n$ and can be expressed as [3], [11], [12]

$$
\mathbf{D}=\left[\begin{array}{cccc}
\sqrt{\lambda_{1}} & 0 & \ldots & 0 \\
0 & \sqrt{\lambda_{2}} & \ldots & 0 \\
\vdots & \vdots & \ddots & 0 \\
0 & 0 & \ldots & \sqrt{\lambda_{l}}
\end{array}\right]
$$

where $\lambda_{1}, \ldots, \lambda_{l}, l=\min (m, n)$ are the eigenvalues of $\mathbf{H H}^{*}$. Taking the definition [3], [9]

$$
\begin{aligned}
& \mathbf{y}=\mathbf{U} \cdot \tilde{\mathbf{y}} \\
& \mathbf{S}=\mathbf{V} \cdot \widetilde{\mathbf{s}} \\
& \mathbf{n}=\mathbf{U} \cdot \tilde{\mathbf{n}}
\end{aligned}
$$

and substituting (4) and (6) into (3), the received signal can be stated as

$$
\tilde{\mathbf{y}}=\mathbf{D} \cdot \widetilde{\mathbf{s}}+\tilde{\mathbf{n}}
$$

Equation (7) represents the MIMO system as $m$ equivalent parallel SingularInput Singular-Output (SISO) channels with the signal powers given by $\sqrt{\lambda_{1}}, \ldots, \sqrt{\lambda_{m}}$. 
A special signal processing is needed at the receiver side to unmix the data streams. The V-BLAST demodulator extracts $\mathbf{s}$ from $\mathbf{y}$ using iterative nulling and cancellation based on a Zero Forcing (ZF) criterion.

In the first step of the demodulation process, a decision statistic is used as a threshold to estimate the received signal. When ZF criterion is used for nulling, the decision statistic $\mathbf{r}$ to determine $\mathbf{s}$ is [2]

$$
\mathbf{r}=\mathbf{w}^{T} \mathbf{y}
$$

where $\mathbf{w}^{T}$ is the transpose of $\mathbf{w}$, which is the weighting vector that satisfies [2]

$$
\mathbf{w}^{T}\left(\mathbf{U} \cdot \mathbf{D} \cdot \mathbf{V}^{*}\right)=\mathbf{I}
$$

with I as an identity matrix. Subtituting (3) and (9) into (8) will yield

$$
\mathbf{r}=\mathbf{S}+\mathbf{w}^{T} \mathbf{n}
$$

The quantization of $\mathbf{r}$ will yield $\mathbf{s}$ and therefore the transmitted symbols are successfully extracted from the received signal $\mathbf{y}$. However as the signals are mixed in the receiver block, a cancellation process is done to detect a particular signal. This cancellation process will take one particular sub-stream of information, and the other sub-streams are assumed to be 'interferers'. Hence, these 'interferers' are cancelled out from the received signals. For example, to detect $y_{1}$ from $\mathbf{y}$, the symbols $y_{2}, y_{3}, \ldots, y_{m}$ are considered interferers and cancelled out from $\mathbf{y}$. The cancellation process can be stated as

$$
\begin{aligned}
y_{i}^{\prime} & =\mathbf{y}-\sum_{j=1}^{i-1} \mathbf{s}_{j} \\
& =\mathbf{U} \cdot \tilde{\mathbf{y}}-\sum_{j=1}^{i-1} \sqrt{\lambda_{j}} \tilde{s}_{j}
\end{aligned}
$$

\subsection{System Performance}

The system performance can be measured as the number of error bits in the receiver, which can be defined as the occurrence of $\left(y_{i} \in \mathbf{y} \neq s_{i} \in \mathbf{s}\right)$. To analytically measure the system performance, the probability that the detected symbol is not equal to the transmitted symbol, $\mathrm{P}\left(y_{i} \neq s_{i}\right)$, has to be stated. $\mathrm{P}\left(y_{i} \neq\right.$ $s_{i}$ ) is a function of the signal to noise ratio (SNR) at a particular detection step and the probabilities of error both in the demodulation and decoding process, and can be stated as 


$$
P_{b}=\sum_{i=1}^{m} \beta_{i}(\gamma) \mathrm{B}_{i}(\gamma)
$$

where $\gamma$ is the instantaneous signal to noise ratio (SNR), $\beta_{i}(\gamma)$ is the SNR pdf at the $i$-th detection step, and $\mathrm{B}_{i}(\gamma)$ is the instantaneous BER that depends on the modulation type and the coding rate used. For the numerical simulations, the number of transmit antennas is $m=2$. Therefore the detection process is done in two steps.

The SNR pdf for the first and second detection steps are [13]

$$
\begin{aligned}
& \beta_{1}(\gamma)=\frac{\partial}{\partial \gamma} F_{1}\left(\frac{\gamma}{\gamma_{0}}\right) \\
& \beta_{2}(\gamma)=\frac{\partial}{\partial \gamma} F_{2}\left(\frac{2 \gamma}{\gamma_{0}}\right)
\end{aligned}
$$

where $\gamma_{0}$ is the average pre-processing SNR per transmit antenna, which is assumed to be equal for both transmit antennas, and the outage probabilities of the first and second detections steps are [13]

$$
\begin{aligned}
F_{1}\left(\frac{\gamma}{\gamma_{0}}\right) & =1-2 e^{-\frac{\gamma}{\gamma_{0}}}+\left(1+\frac{\gamma}{2 \gamma_{0}}\right) e^{-\frac{2 \gamma}{\gamma_{0}}} \\
F_{2}\left(\frac{\gamma}{\gamma_{0}}\right) & =1-\left[1-F_{h}\left(\frac{\gamma}{\gamma_{0}}\right)\right]^{2} \\
& =F_{h}\left(\frac{\gamma}{\gamma_{0}}\right)\left[2-F_{h}\left(\frac{\gamma}{\gamma_{0}}\right)\right]
\end{aligned}
$$

where the general form of outage probability $F_{h}\left(\frac{\gamma}{\gamma_{0}}\right)$ can be stated as [13]

$$
F_{h}\left(\frac{\gamma}{\gamma_{0}}\right)=1-e^{-\left(\frac{\gamma}{\gamma_{0}}\right)}\left(1+\frac{\gamma}{\gamma_{0}}\right)
$$

In case of a system where the number of transmit antennas is 2 and the number of receive antennas is $n>2$, the outage probabilities can be stated as [13] 


$$
F_{1}\left(\frac{\gamma}{\gamma_{0}}\right)=1-(n-1) e^{-\frac{\gamma}{\gamma_{0}}}\left[\sum_{i=0}^{n-2} 2 a_{i}-e^{-\frac{\gamma}{\gamma_{0}}}\left[b_{i}+\left(2 \frac{\gamma}{\gamma_{0}}\right)^{n-1} c_{i}\left[\frac{\gamma}{\gamma_{0}}\right)^{i}\right]\right.
$$

where [13]

$$
\begin{aligned}
& a_{i}=\sum_{m=0}^{i} \frac{(-1)^{i-m}(n-i-2) !}{(n-m-1) ! m !} \\
& b_{i}=(-2)^{i}(n-i-2) ! \sum_{k=0}^{i} \frac{(-1)^{k}}{k !(n-1-k) !} \\
& c_{i}=\frac{2^{i}}{i !} \sum_{j=i+n k=j-n+11}^{2 n-2} \sum^{n-2} \frac{(j-n) ! 2^{-j}}{k !(j-k) !}
\end{aligned}
$$

The outage probability for the second detection step is given by (16), where $F_{h}\left(\frac{\gamma}{\gamma_{0}}\right)$ for this case is [13]

$$
F_{h}\left(\frac{\gamma}{\gamma_{0}}\right)=1-e^{-\frac{\gamma}{\gamma_{0}}} \sum_{k=0}^{n-1} \frac{\left(\frac{\gamma}{\gamma_{0}}\right)^{k}}{k !}
$$

The instantaneous BER of the system $\mathrm{B}_{i}(\gamma)$ is upperbounded by the Viterbi's upperbound on error event probability [14]. As an RCPC code is used instead of a convolutional code, $\mathrm{B}_{i}(\gamma)$ can be stated as

$$
\mathrm{B}_{i}(\gamma)=\frac{1}{L} \sum_{d=d_{\text {free }}}^{\infty} c_{d} P_{d}
$$

where $c_{d}$ is the the total number of error bits contained in the incorrect paths in the convolutional trellis and $P_{d}$ is the probability of selecting a wrong path at distance $d$. For a Rayleigh fading channel with soft decision on $\mathbf{y}$ and full CSI, $P_{d}$ can be upperbounded by [4]

$$
P_{d} \leq \frac{1}{2}\left(\frac{1}{1+\gamma R_{c}}\right)^{d}
$$




\section{$3 \quad$ Simulation Results}

For the simulation we use $m=2$, encoder memory $M=5$ and puncturing periods $L=5$ and 6. In the first simulation the code rates used are $R_{c 1}=5 / 15$ and $R_{c 2}=$ $5 / 10$ for the first and the second detected data stream, respectively. The lower code rate is given to a data stream entering a non-destructive sub-channel. In the second simulation, the code rates used are $R_{c 1}=6 / 18$ and $R_{c 2}=6 / 12$ for the data stream entering a non-destructive and a destructive sub-channel, respectively.

For the first simulation we use encoders A, B, C and D depicted in Table 1. The simulation result is given in Figure 3. Encoder A provides $R_{c 1}=5 / 15$ and therefore gives a high level of protection intended for the data stream of information entering a sub-channel with low attenuation. Encoders B, C and D provide $R_{c 2}=5 / 10$ for the other data streams of information that enter a subchannel with high attenuation. It is shown that the different puncturing matrices used in encoders B, C and D give similar performances. In fact, the performances yielded by encoders $\mathrm{C}$ and $\mathrm{D}$ almost match each other. This is due to their equal $d_{\text {free }}=6$ and comparable $c_{d}$ values. It is also shown that with a single encoder and decoder structure, the proposed system can achieve up to 2 $\mathrm{dB}$ coding gain by increasing the code rate. Hence for a sub-channel with high attenuation, $R_{c 2}$ can be used to save $2 \mathrm{~dB}$ of bandwidth requirement at the expense of lower performance.

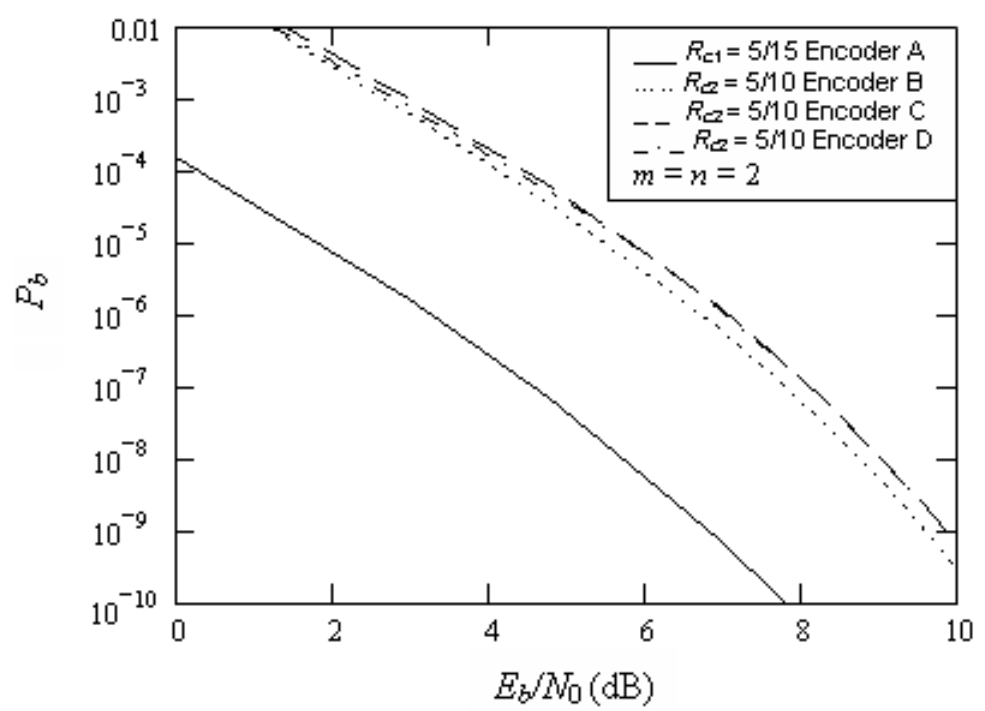

Figure 3 Results for RCPC-encoded V-BLAST MIMO using encoders A, B, C and D. 


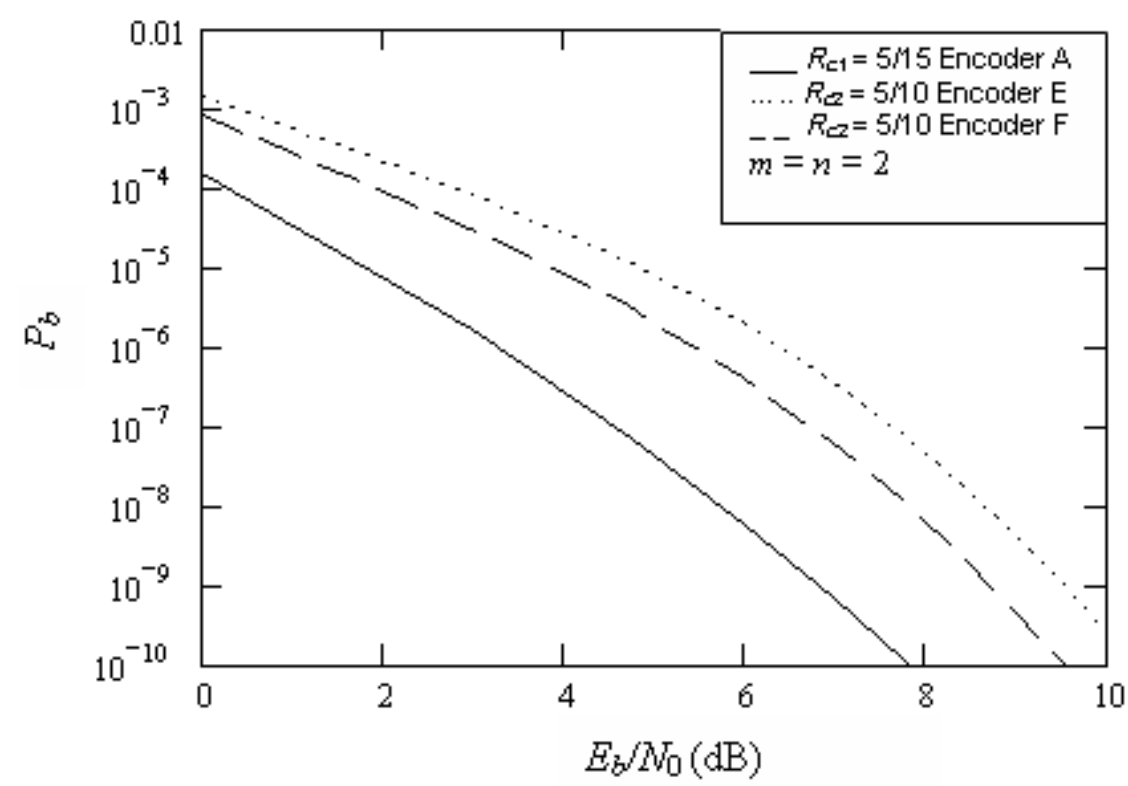

Figure 4 Results for RCPC-encoded V-BLAST MIMO using encoders A, E and $\mathrm{F}$.

The system performance yielded by encoders $\mathrm{E}$ and $\mathrm{F}$ are given in Figure 4. In these encoders the 5 punctured bits are dispersed, 4 bits in one row and an additional bit in another row. Puncturing is done only to the least significant bits, leaving the most significant bits (the bits in the left-most side) intact. Although the maximum gain variation of $2 \mathrm{~dB}$ is still retained, encoders $\mathrm{E}$ and $\mathrm{F}$ yield better performances compared to encoders B, C and D. This is due to the fact that puncturing 5 bits in a row essentially means inactivating one summing point in the encoder thus degrading the performance noticeably.

The system performances of encoders $\mathrm{H}$ and $\mathrm{I}$ are given in Figure 5. The punctured bits are also dispersed in two rows for these encoders, and the results are similar to the ones shown in Figure 4. This implies that dispersing the punctured bits in a varied manner will yield similar performance. It is noted however that puncturing the most significant bits will not enable us to attain equivalent generator polynomials, therefore we do not include puncturing matrices where the most significant bits are punctured. 


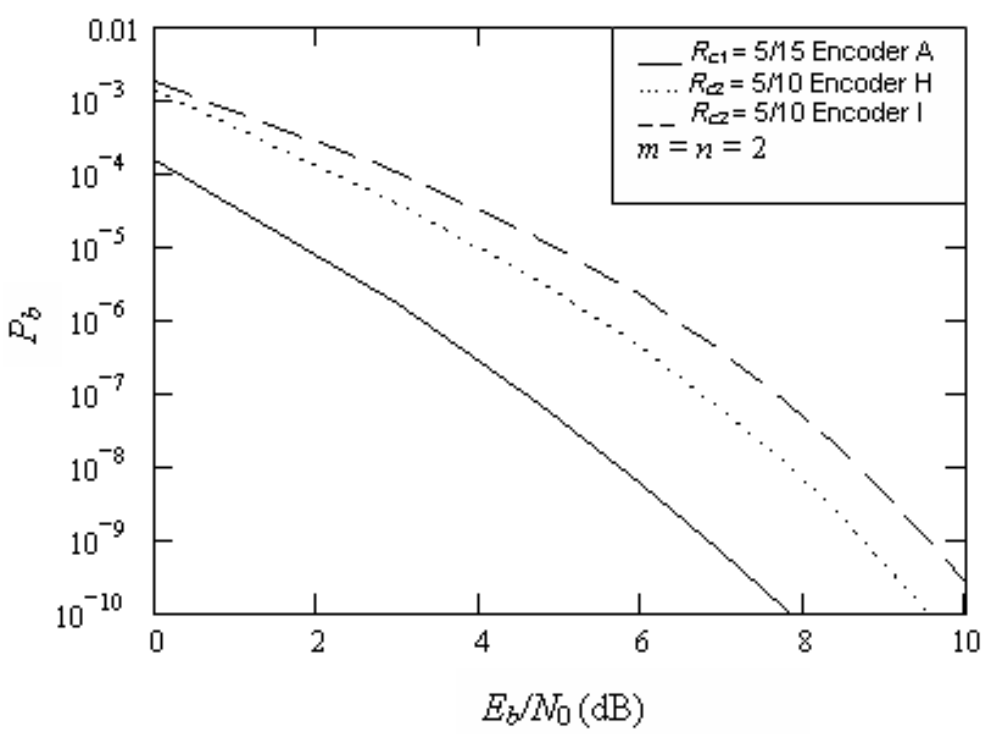

Figure 5 Results for RCPC-encoded V-BLAST MIMO using encoders A, H and I.

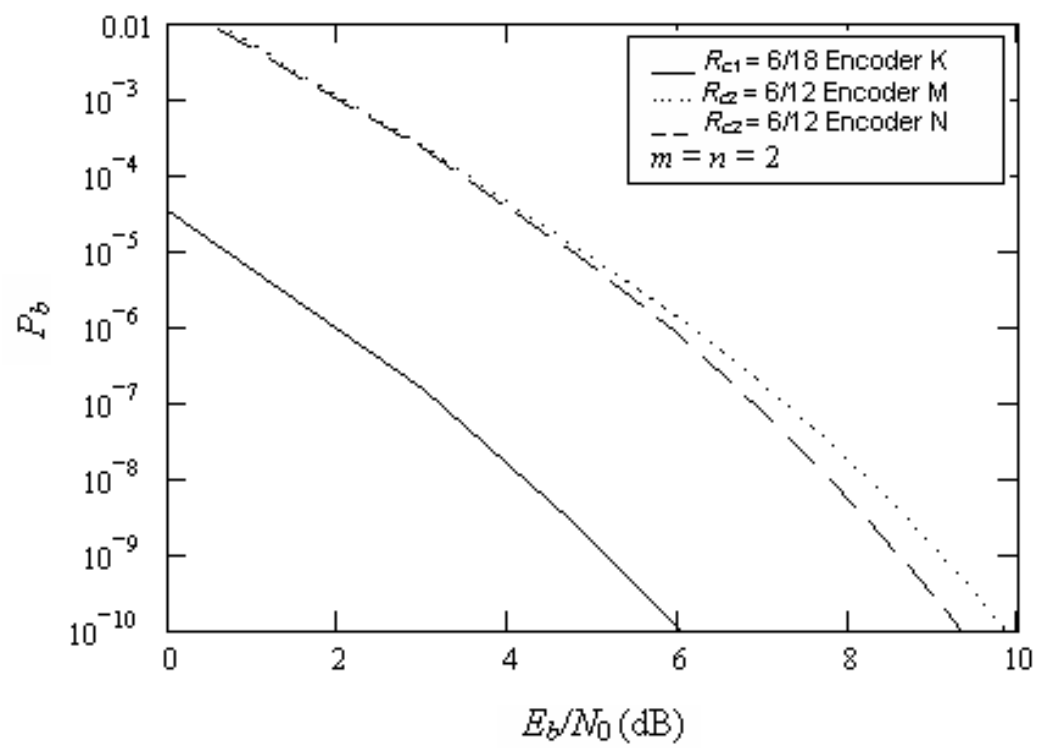

Figure 6 Results for RCPC-encoded V-BLAST MIMO using encoders K, M and $\mathrm{N}$. 


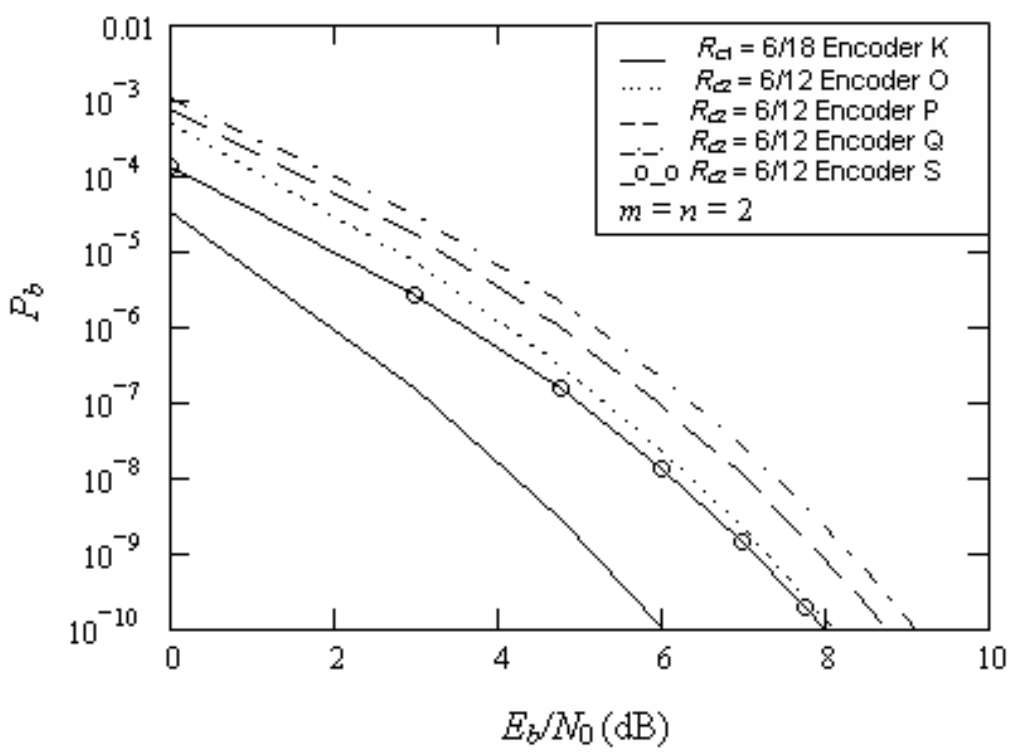

Figure 7 Results for RCPC-encoded V-BLAST MIMO using encoders K, O, P, $\mathrm{Q}$ and $\mathrm{S}$.

The simulation results of RCPC codes with puncturing period $\mathrm{L}=6$ are given in Figures 6 and 7. The encoders used are listed in Table 2. It is shown that the system with $\mathrm{L}=6$ outperforms the system with $\mathrm{L}=5$. This is due to the increased $\mathrm{d}_{\text {free }}$ attained by increasing the puncturing period. The gain variation attained by using encoders $\mathrm{K}, \mathrm{M}$, and $\mathrm{N}$ is higher than the variation attained by using encoders $\mathrm{O}, \mathrm{P}, \mathrm{Q}$ and $\mathrm{S}$. In general it is shown that large gain variation is attainable by choosing a large puncturing period $\mathrm{L}$. As in the case of $\mathrm{L}=5$, the dispersed punctured bits yield better performance compared to puncturing bits in one row. It is also further confirmed that dispersing the punctured bits in a varied manner will yield similar performances.

The numerical results thus far have shown that increasing the code rate by $33.3 \%$ from $R_{c 1}=5 / 15$ or $6 / 18$ to $R_{c 2}=6 / 10$ or $6 / 12$ causes the system performance to decrease. This increase in the code rate also means that the bandwidth efficiency is increased by 33.3\%. We note that the best performing code is given by puncturing matrix $\mathrm{S}$, where increasing the code rate by $33.3 \%$ from $R_{c 1}=6 / 18$ to $R_{c 2} 6 / 12$, at the cost of decreasing the system performance by approximately $2 \mathrm{~dB}$. However this penalty is not severe because $R_{c 2}=6 / 12$ will only be used in sub-channels where the attenuation gain is high. 
As the puncturing period $L=6$ improves the system performance compared to $L$ $=5$, in the next numerical simulations we use $L=6$ for a system with $m=2$ and $n=4$. The parameter $n=4$ is chosen because in $3 \mathrm{GPP}$ it has been stated that advanced MIMO techniques could employ up to 4 receive antennas, while keeping the codewords transmitted to 2 layers [15].

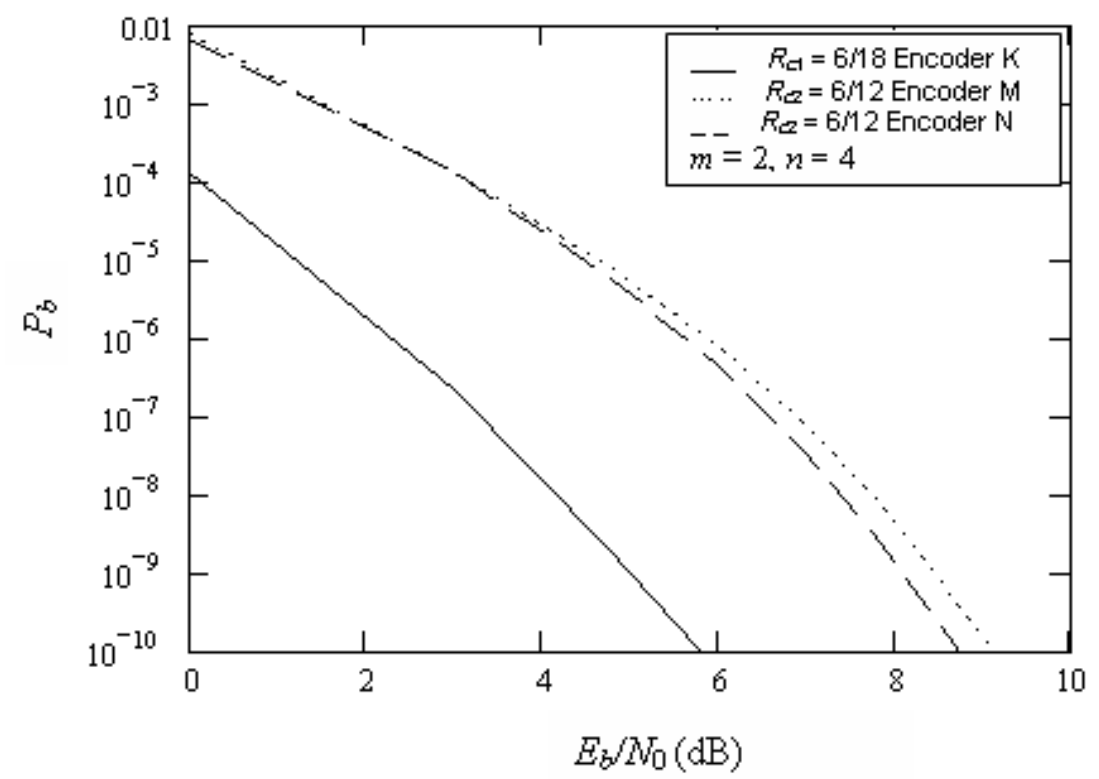

Figure 8 Results for RCPC-encoded V-BLAST MIMO using encoders K, M, and $\mathrm{N}$ with $m=2$ and $n=4$.

The system performance for encoders $\mathrm{K}, \mathrm{M}$, and $\mathrm{N}$ is depicted in Figure 8 . Compared to the system with $m=n=2$, the performance improvement is within $1 \mathrm{~dB}$ for a BER level of $10^{-10}$. The result of further simulation using encoders $\mathrm{K}, \mathrm{O}, \mathrm{P}, \mathrm{Q}$ and $\mathrm{S}$ for a system with $m=2$ and $n=4$ is given in Figure 9. It is shown that the addition of receive antennas in this system also only improves the system performance within $1 \mathrm{~dB}$ range for a BER level of $10^{-10}$. These results are in accordance to [13] which states that optimal ordering implemented in V-BLAST MIMO would not contribute to the diversity gain of the system, yet it improves the post-processing SNR for each detection step. Consistent with the results depicted in Figures 3-7, the dispersion of punctured bits will slightly improve the performance of systems with additional receive antennas. It is also noted that for systems with $n=2$ and $n=4$, the dispersion of punctured bits will improve the system performance in the low $E_{b} / N_{o}$ region. 


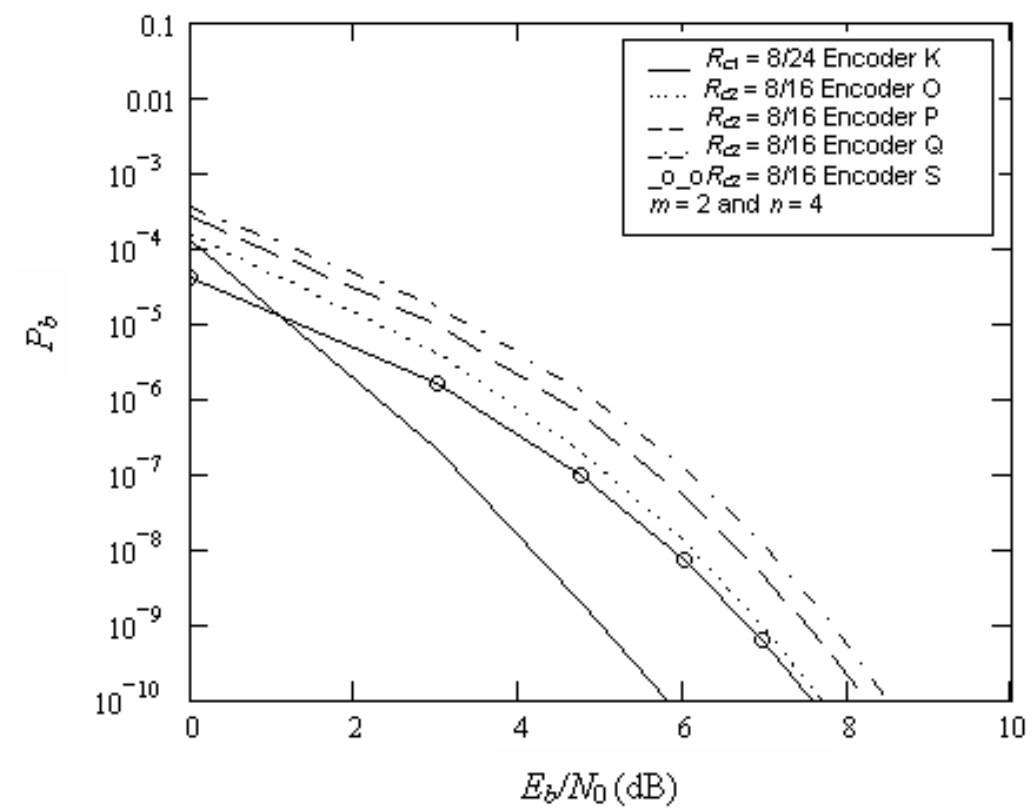

Figure 9 Results for RCPC-encoded V-BLAST MIMO using encoders K, O, P, $\mathrm{Q}$ and $\mathrm{S}$ with $m=2$ and $n=4$.

\section{Conclusion}

An RCPC-encoded V-BLAST MIMO system with $m$ transmit and $n$ receive antennas have been proposed and simulated. Simulation results show that for a BER level of less than $10^{-7}$ the single encoder and decoder structure can provide users with close to $4 \mathrm{~dB}$ gain variation. Thus RCPC-encoded V-BLAST allows for bandwidth preservation by increasing the code rate, at the expense of system performance. Numerical simulations show that a 33.3\% bandwidth efficiency can be attained by increasing the code rate, from $5 / 15$ or $6 / 18$ to $5 / 10$ and $6 / 12$, respectively. This increase in bandwidth efficiency will decrease the system performance. However as the increased code rate is utilized for the data stream entering a sub-channel with high attenuation, this disadvantage is not severe. It is also shown that in general dispersing the punctured bits will yield better performances as opposed to puncturing more than one whole row of data stream, although the code rate is maintained. Further, it is shown that additional receive antennas $(n=4)$ would improve the system performance within $1 \mathrm{~dB}$ range compared to a system with $n=2$. Therefore, the performance improvement provided by the additional antennas might not be significant enough in light of the increased cost and complexity they introduce. As the puncturing matrices used in the RCPC codes evidently affect the system 
performance, further researches regarding the optimal puncturing matrices for an RCPC-encoded V-BLAST MIMO system are needed.

\section{Reference}

[1] Foschini, G.J, Layered Space-time Architecture for Wireless Communication in a Fading Environment when Using Multi-Element Antenna, Bell Laboratories Technical Journal, 41-59, 1996.

[2] Wolniansky, P.W., et al, V-BLAST: An Architecture For Realizing Very High Data Rates Over The Rich-Scattering Wireless Channel, in URSI International Symposium on Signals, Systems and Electronics, pp. 295300, 1998.

[3] Kulakowski, P., The Multiple-Input Multiple-Output Systems in Slow and Fast Varying Radio Channels, Ph.D dissertation, Department of Telecommunication, AGH University of Science and Technology, Krakow, 2007.

[4] Hagenauer, J., Rate-Compatible Punctured Convolutional Codes (RCPC Codes) And Their Applications, IEEE Trans. on Communications, 8(4), 389-400, 1988.

[5] Farooq, M.S., et al., An Unequal Error Protection Scheme For Multiple Input Multiple Output Systems, in $36^{\text {th }}$ Asilomar Conference on Signals, Systems and Computers, pp 575-579, 2002.

[6] Guo, R., et al., BER Performance Analysis Of RCPC Encoded MIMOOFDM In Nakagami-M Channels, in International Conference on Wireless Communications, Networking \& Mobile Computing, pp 1416 1420, 2006.

[7] Noh, Y., et al., Design of Unequal Error Protection For MIMO-OFDM Systems, in $61^{\text {st }}$ IEEE Vehicular Technology Conference, pp. $1058-$ $1062,2005$.

[8] Yang, G., et al., Unequal Error Protection for MIMO Systems With A Hybrid Structure, in IEEE International Symposium on Circuits and Systems, pp. 682-685, 2006.

[9] Sari, L., Wibisono, G. \& Gunawan, D. BER Performance Analysis of VBLAST MIMO System with Joint Source and Channel Coding, in IEEE Pacific-Asia Workshop on Computational Intelligence and Industrial Application, pp. 733-737, 2008.

[10] Sari, L., Wibisono, G. \& Gunawan, D. An Enhanced V-BLAST MIMO System Using Joint Source and Channel Coding, in Proc. IEEE Int. Conf. On Advanced Communication Tech. (ICACT 09), pp. 635-639, 2009.

[11] Gesbert, D., et al., From Theory To Practice: An Overview of MIMO Space-Time Coded Wireless Systems, IEEE Journal on Selected Areas in Communications, 21(3), 281-302, 2003. 
[12] Wenstrom, M., On MIMO Systems and Adaptive Arrays for Wireless Communication: Analysis and Practical Issues, Ph.D dissertation, Department of Material Science, Uppsala University, Stockholm, 2002.

[13] Loyka, S. \& Gagnon, F., Performance Analysis Of The V-BLAST Algorithm: An Analytical Approach, IEEE Trans. on Wireless Communications, 3(4), pp. 1326-1337, 2004

[14] Viterbi, A.J. \& Omura J.K., Principles of Digital Communication and Coding, McGraw Hill, 1979.

[15] Virtej, E., Kuusela, M. \& Tuomaala, E. System Performance of SingleUser MIMO in LTE Downlink, in $19^{\text {th }}$ IEEE Personal, Indoor and Mobile Radio Conference, pp. 1-5 , 2008. 